\title{
Estudo comparativo do acesso semântico no processamento visual de palavras entre brasileiros monolíngues e chineses multilíngues falantes do português do Brasil como língua estrangeira
}

\section{Comparative study of the semantic access in the visual processing of words between monolinguistic Brazilians and multilinguistic Chinese who speak Portuguese as a foreign language}

\author{
Jerusa Fumagalli de Salles ${ }^{1}$ \\ Candice Steffen Holderbaum ${ }^{2}$ \\ Ingrid Finger ${ }^{3}$
}

\begin{abstract}
RESUMO
O priming semântico é uma forma de avaliar o processamento semântico de palavras. Se a semântica é um importante fator contribuinte no reconhecimento visual de palavras, surge a questão de se chineses multilíngues (mandarin como L1 e inglês como L2), que estão aprendendo o português como L3, podem se beneficiar do contexto semântico em tarefa de decisão lexical na Língua Portuguesa, comparado aos controles (brasileiros universitários e crianças). Além de comparar a magnitude do efeito de priming semântico entre a amostra de chineses e de brasileiros, objetivou-se investigar nos chineses a relação entre o desempenho no experimento de priming semântico e na tarefa de consciência fonológica, ambos na língua portuguesa. Participaram do estudo 40 universitários chineses multilíngues, 31 universitários brasileiros e 26 crianças
\end{abstract}

1 Professora Adjunta do Instituto de Psicologia, Programa de pós-graduação em Psicologia da Universidade Federal do Rio Grande do Sul - Brasil. E-mail: jerusafs@yahoo.com; www.ufrgs.br/neuropsicologia

2 Doutoranda do Programa de Pós Graduação em Psicologia da Universidade Federal do Rio Grande do Sul- Brasil.

3 Professora Adjunta do Instituto de Letras, Programa de pós-graduação em Letras da Universidade Federal do Rio Grande do Sul - Brasil. 
de $3^{\text {a }}$ série. Houve efeito de priming semântico nos chineses e nos brasileiros, universitários e crianças, ou seja, respostas mais rápidas na condição com prime relacionado do que na condição com prime não relacionado. Não houve diferenças significativas na magnitude do efeito entre os grupos de adultos, mas as crianças apresentaram maior magnitude de efeito do que os chineses. Considerando apenas o grupo de chineses, não houve correlação entre os escores na tarefa de decisão lexical no paradigma de priming semântico e a avaliação da consciência fonológica. Chineses parecem ter acessado o significado dos primes apresentados visualmente na Língua Portuguesa, não se diferenciando dos brasileiros adultos e crianças.

Palavras-chave: processamento semântico; priming semântico; decisão lexical; multilinguismo; consciência fonológica.

\begin{abstract}
The semantic priming paradigm can be used to evaluate word semantic processing. Considering that semantic is an important factor in visual word recognition, an experiment was conducted to verify if multilingual Chinese (L1 and L2 being respectively Mandarin and English) that are learning Portuguese as L3 would benefit from the semantic context during a lexical decision task with stimuli presented in Portuguese. The magnitude of the semantic priming effect was compared to two control groups (Brazilian children and adults). Moreover, this study aimed to investigate the relation between the performance in the lexical decision task and the one in the Portuguese phonological awareness task in the Chinese group. The sample was composed of 40 undergraduate multilingual Chinese, 31 undergraduate Brazilians and 26 third-grade children. Results showed semantic priming effects in all groups, which means that reaction time was smaller when a related prime preceded the target. No significant difference was found regarding the magnitude of the semantic priming effects between Chinese and Brazilian adults. However, such a difference appeared between the Chinese adults and the Brazilian children group. Finally, no correlation was found between lexical decision performance and score at the phonological awareness task. We concluded that Chinese, like both Brazilians groups tested, seem to access the meaning of presented primes.
\end{abstract}

Keywords: semantic processing; semantic priming; lexical decision; multilingualism; phonological awareness.

\title{
Introdução
}

O paradigma de priming semântico tem sido utilizado nos estudos de reconhecimento visual de palavras (leitura) para investigar o acesso à informação 
semântica, especialmente com amostras de leitores proficientes, adultos, universitários, monolíngues, e nas línguas inglesa, francesa ou espanhola (SALLES; JOU; STEIN, 2008). Os estudos com bilíngues ou multilíngues têm sido mais raros e foram realizados especialmente nas últimas duas décadas (ex: FINKBEINER, 2003; FINKBEINER; FORSTER; NICOL; NAKAMURA, 2004; GOLLAN; FORSTER; FROST, 1997; JIANG, 1999; JIANG; FORSTER, 2001) e, em geral, contêm estímulos em ambas as línguas, paradigma conhecido como "crosslanguage translation priming" (ALTARRIBA; BASNIGHT-BROWN, 2007; KEATLEY; SPINKS; GELDER, 1994).

As definições de bilinguismo/multilinguismo variam de acordo com critérios relacionados aos níveis de proficiência e aos diferentes modos pelos quais um indivíduo pode vir a se tornar bilíngue/multilíngue. Segundo Bloomfield (1933), por exemplo, os bilíngues são indivíduos que possuem controle nativo de duas línguas. De forma semelhante, Saer (1922), em sua Hipótese do Duplo Monolíngue, defendeu que o indivíduo bilíngue reúne características de dois sujeitos monolíngues em uma única pessoa, devendo ter desempenho equivalente, nas duas línguas, aos falantes monolíngues de cada uma delas. Essa concepção tem sido bastante criticada por autores como Grosjean (1989), para quem o modo de ativação e processamento das línguas constitui um continuum, de um modo monolíngue a um modo bilíngue, passando por vários estados intermediários de processamento e ativação das línguas usadas. O perfil do bi/multilíngue muda à medida que ele avança no continuum ou deixa de utilizar uma das línguas. Neste artigo, compreende-se o multilinguismo como a habilidade de utilizar mais do que duas línguas, a partir de uma concepção de que bilíngues e multilíngues são indivíduos que possuem níveis de competência e de fluência diferentes nas línguas que usam.

O acesso semântico no processamento visual de palavras em monolíngues e em bilíngues/multilíngues pode ter características distintas em função do grau de proficiência na língua na qual a tarefa é realizada. O priming semântico, uma forma de avaliar o processamento semântico de palavras na leitura, está inserido no fenômeno de Priming, ou seja, um tipo de memória implícita (não declarativa) referente aos efeitos facilitadores de eventos antecedentes (primes) sobre o desempenho subsequente (alvos) (FORSTER, 1999). Quando esta facilitação ocorre devido a uma relação semântica existente entre prime e alvo, esse efeito é denominado priming semântico. No paradigma típico de priming semântico, mede-se a acurácia e velocidade de resposta à palavra alvo, sendo que algumas vezes ela é precedida ou ativada por uma palavra ou sentença semanticamente relacionada e, em outras, condições controle, ela é precedida por uma palavra ou sentença não relacionada (NIEVAS; JUSTICIA, 2004).

Esse paradigma metodológico permite estudar experimentalmente o acesso ao léxico mental e a influência do contexto semântico no reconhecimento de 
palavras lidas. A tarefa de decisão lexical (decidir, o mais rapidamente possível, se o alvo é uma palavra ou uma pseudopalavra) não requer que o participante leia em voz alta as palavras, ou seja, não penaliza os participantes com dificuldades de leitura oral (BETJEMANN; KEENAN, 2008). Além disso, este paradigma permite estudar o acesso semântico no reconhecimento visual de palavras de forma on-line, durante o processo e de forma indireta.

Em uma abordagem neuropsicológica cognitiva, os processos cognitivos envolvidos na leitura competente, considerando o processamento de palavras, variam conforme o modelo teórico, mas todos perpassam os níveis fonológico, ortográfico e semântico. Em modelos de leitura de Processamento Paralelo Distribuído (PPD) (ou conexionistas), como o de Plaut, McClelland, Seidenberg e Patterson (1996), as informações semânticas, fonológicas e ortográficas atuam na identificação de palavras escritas. Essas informações são representadas em termos de padrões distribuídos de atividade em grupos distintos de unidades de processamento, semelhantes a neurônios (PLAUT et al., 1996; SEIDENBERG; MCCLELLAND, 1989). Dessa forma, a informação semântica também facilita o reconhecimento de palavras e torna a leitura mais eficiente, embora, de modo geral, os aspectos semânticos do processamento de leitura têm sido negligenciados nos estudos de leitura.

Se a semântica é um importante fator contribuinte no reconhecimento visual de palavras, surge a questão de se chineses multilíngues (mandarin como L1 primeira língua - e inglês como L2 - segunda língua), que estão aprendendo o português como L3 (terceira língua), podem se beneficiar do contexto semântico no reconhecimento visual de palavras (leitura) em uma tarefa de decisão lexical na língua portuguesa, comparado aos controles (brasileiros, universitários e crianças de $3^{a}$ série, que tem o português como língua materna). Como os chineses multilíngues estão aprendendo a ler em português (como L3), eles tiveram, portanto, menos exposição às informações ortográficas e semânticas das palavras na língua portuguesa (léxico escrito), comparado aos brasileiros universitários, o que poderia refletir-se em representações semânticas e conexões menos desenvolvidas. Por outro lado, eles podem compensar a menor proficiência no reconhecimento visual de palavras com o uso da informação contextual, nesse caso, semântica. Outro questionamento aliado a esse é se o escore em tarefa de consciência fonológica no português estaria relacionado ao desempenho na tarefa de decisão lexical no paradigma de priming semântico na amostra de chineses.

Especialmente nas línguas alfabéticas ${ }^{4}$, a consciência fonológica tem uma estreita relação com a aprendizagem da leitura (MORAIS, 1985; STAHL; MURRAY,

4 As unidades do sistema alfabético de escrita são, por natureza, sublexicais e referem-se aos "sons" da língua falada. 
1994). A hipótese mais aceita é a de que haja uma relação recíproca entre consciência fonológica e leitura, ou seja, aprender a ler ocasiona o desenvolvimento das habilidades de consciência fonológica e estas, por sua vez, facilitam a aprendizagem da leitura (STACKHOUSE, 1997). A consciência fonológica, consciência dos sons em palavras faladas, é avaliada por tarefas de rima, comparação de consoantes iniciais (aliteração), contagem do número de fonemas em palavras faladas (STAHL; MURRAY, 1994), adição, subtração e inversão de sílabas ou fonemas. No nível de maior dificuldade linguística, a consciência fonológica envolve a habilidade de reconhecer e manipular fonemas (consciência fonêmica). Anthony e Lonigan (2004) defendem que a consciência fonológica é formada por várias habilidades altamente correlacionadas, que podem ser medidas por uma variedade de tarefas que diferem em complexidade linguística (sílabas, rimas, onsets - equivalentes a consoante inicial da sílaba - e fonemas).

Para os chineses, a consciência fonêmica pode ter uma utilidade relativamente limitada para a aprendizagem inicial da leitura na língua materna (MCBRIDE-CHANG et al., 2005). No estudo de McBride-Chang et al. (2005), com crianças chinesas de segunda série, tarefas de consciência da estrutura morfológica foram preditoras do desempenho em leitura em chinês, mas as tarefas de consciência fonológica não. Na China, o mandarin é a língua falada e o "pinyin" é um sistema de codificação fonológica ensinado às crianças no início do processo de alfabetização. Uma representação "pinyin" de cada novo caractere chinês é pareada quando o reconhecimento de palavras é ensinado (MCBRIDE-CHANG et al., 2005). Como no chinês cada caractere representa uma única sílaba, a consciência de sílaba e de rima são medidas confiáveis de consciência fonológica nessa língua (SIOK; FLETCHER, 2001, apud MCBRIDE-CHANG et al., 2005). Em termos de desenvolvimento, Suk-Han Ho e Bryant (1997) encontraram que apesar de as crianças chinesas desenvolverem a consciência de onsets e rimes, isso ocorre bem mais tarde quando comparado a crianças falantes do inglês.

Com relação ao processamento semântico de palavras avaliado pelo experimento de priming semântico, foram encontrados poucos estudos empíricos com bilíngues e multilíngues avaliando-se apenas na segunda ou terceira língua. A maioria dos estudos é de priming em duas línguas, L1 e L2 (por exemplo: FINKBEINER, 2003; FINKBEINER; FORSTER; NICOL; NAKAMURA, 2004; GOLLAN; FORSTER; FROST, 1997; JIANG, 1999; JIANG; FORSTER, 2001). O uso do paradigma de priming semântico com estímulos em duas línguas tem sido utilizado para compreender como bilíngues armazenam duas línguas na memória (ALTARRIBA; BASNIGHT-BROW, 2007).

Questões metodológicas podem influenciar os efeitos de priming semântico, como, por exemplo, a proficiência na língua. Alguns estudos têm sugerido que 
bilíngues mais proficientes apresentam maiores efeitos de priming do que aqueles menos proficientes em sua segunda língua (L2) (ALTARRIBA; BASNIGHTBROW, 2007). No entanto, outros estudos têm mostrado efeitos de priming semântico não significativos para bilíngues mais proficientes e efeitos significativos para bilíngues menos proficientes (FRENCK; PYNTE, 1987). No presente estudo avaliou-se apenas os estímulos na Língua Portuguesa, ou seja, terceira língua aprendida pelo chineses, já na idade adulta.

Martin e colaboradores (2009) avaliaram bilíngues (inglês e galês) adultos, que aprenderam as duas línguas desde pequenos, em uma tarefa de contagem de letras em palavras. Os estímulos eram apresentados em pares que variavam na língua do prime e do alvo (inglês e galês) e no contexto (prime relacionado e não relacionado). Os resultados mostraram efeitos de priming semântico para alvos em ambas as línguas. Segundo os autores, isso sugere que o acesso ao significado de palavras escritas é automático nas duas línguas para bilíngues que as aprenderam quando pequenos.

Estudos que avaliaram bilíngues em tarefas de decisão lexical utilizando somente estímulos da L2 (por exemplo: GOLLAN et al., 1997; FINKBEINER, 2003), como propõe o presente estudo, utilizam o paradigma de masked priming de repetição (priming não visível), no qual os pares prime-alvo são estímulos iguais (condição experimental) ou diferentes. No estudo de Gollan et al. (1997), os participantes, que apresentavam hebreu como L1 e inglês como L2, realizaram tarefa de decisão lexical com estímulos em inglês (experimento 1) e em hebreu (experimento 2), com prime apresentado por $50 \mathrm{~ms}$ (geralmente insuficiente para processamento consciente) e alvo por $500 \mathrm{~ms}$. Os resultados mostraram efeito de priming de repetição, ou seja, respostas mais rápidas quando o prime e o alvo eram iguais, tanto no inglês quanto no hebreu. Finkbeiner (2003), utilizando experimento semelhante ao usado por Gollan et al. (1997), com japoneses bilíngues que possuíam o inglês como L2, também encontrou efeito de priming de repetição para estímulos na L2. Segundo os autores, os resultados são indicativos de um processamento automático semelhante ao demonstrado por participantes avaliados em suas línguas nativas.

O presente trabalho teve, portanto, como objetivo comparar a magnitude dos efeitos de priming semântico entre universitários chineses multilíngues (português como L3) e brasileiros (adultos e crianças) falantes do português em uma tarefa de decisão lexical com estímulos em português. Questiona-se se nos participantes chineses a apresentação de primes semanticamente relacionados aos alvos facilita a decisão lexical dos alvos, comparado a dois grupos de brasileiros, um deles emparelhado aos chineses pelo nível de escolaridade e idade (adultos universitários) e outro grupo formado por crianças que, como os chineses, estão em processo de aprendizagem da leitura ( 2 anos de ensino 
formal da língua escrita). Pretendeu-se, também, analisar as correlações entre os escores na tarefa de decisão lexical no paradigma de priming semântico e a avaliação de consciência fonológica no grupo de chineses.

\section{Método}

\section{Participantes}

Participaram do estudo 40 universitários chineses multilíngues com mandarim ou dialeto como L1, inglês como L2 e português como L3, com média de idade 21,16 anos. Esse grupo foi comparado a dois grupos de brasileiros: 1) 26 crianças de $3^{\text {a }}$ série do Ensino Fundamental com idade entre 8 e 9 anos (média de idade $=8,35$ anos e DP $=0,45$ ), e 2) 31 universitários brasileiros com idade entre 17 e 38 anos (média de idade $=21,06$ e DP $=2,61$ ) de uma universidade pública do Rio Grande do Sul. Os universitários chineses moravam no Brasil há um ano, participando de intercâmbio, tendo sido aprovados no CELPEBRAS. Eles começaram a aprender o português escrito antes de vir ao Brasil, tendo estudado por 2 anos completos na Universidade de origem, por 20 horas semanais. Todos relataram terem iniciado o aprendizado de inglês antes dos 13 anos de idade, com exposição de 2 a 10 horas semanais.

\section{Delineamento, instrumentos e procedimentos}

Trata-se de um delineamento misto, quase experimental, de comparação entre grupos, e correlacional. As variáveis independentes são tipo de prime (semanticamente relacionado ao alvo e não relacionado) e o grupo (chineses, brasileiros universitários e crianças de $3^{\mathrm{a}}$ série). As variáveis dependentes foram tempo de reação (TR) e acurácia da resposta (porcentagem de erros) e a magnitude do efeito de priming semântico.

O instrumento de avaliação do efeito de priming semântico (HOLDERBAUM; SALLES, submetido) consiste em 78 pares de palavras (prime - alvo), metade formada por palavra real (prime) - palavra real (alvo) e a outra metade por palavra real (prime) - pseudopalavra (alvo). Os estímulos foram retirados de um estudo prévio de normas de associação semântica realizado com 154 crianças de $3^{\mathrm{a}}$ série (SALLES; MACHADO; HOLDERBAUM, 2009). Os alvos eram palavras frequentes do vocabulário das crianças de $3^{\mathrm{a}}$ série e tinham no máximo seis letras. Metade desses foram precedidos por primes semanticamente rela- 
cionados (força da associação igual ou maior que 25\%) e metade por primes não relacionados. Os primes não relacionados semanticamente aos alvos foram escolhidos dentro dos dados do mesmo estudo. As pseudopalavras, formadas através da substituição de algumas letras das palavras alvos, mantiveram o mesmo número de letras. Os primes que precederam as pseudopalavras foram palavras ditas por apenas uma criança (respostas idiossincráticas) no estudo de Salles et al. (2009). Alguns exemplos de pares relacionados: aberto - fechado; fácil - difícil; sapo - pular; noite - dia; faca - garfo.

Os 78 pares de palavras do instrumento de avaliação de priming semântico foram apresentados na tela do computador através do software E-PRIME. Para cada par de palavras o participante lia, de forma silenciosa, a primeira palavra (prime) e então decidia se a segunda palavra (alvo) era uma palavra real, apertando na tecla "SIM", ou se era uma pseudopalavra, apertando na tecla "NÃO". O participante era instruído a realizar a tarefa o mais rápido e acuradamente possível. O SOA (Stimulus Onset Assynchrony) ou intervalo entre a apresentação do prime e a do alvo foi de $500 \mathrm{~ms}$. Antes de iniciar o experimento, os participantes receberam as seguintes instruções: "Você deve prestar atenção a cada estímulo que aparece na tela do computador. Tente ler em silêncio a primeira palavra (apresentada em letras minúsculas). Depois disso, uma cruz $(+)$ aparecerá na tela, o que significa que a próxima palavra será apresentada. Você deve decidir, da maneira mais rápida e precisa quanto possível, se esta segunda palavra (apresentada em letras maiúsculas) é uma palavra real, pressionando o botão 'Sim', ou uma palavra que não existe, apertando o botão 'Não"'. Quando o participante terminava de ler as instruções, o pesquisador explicava novamente para garantir a compreensão da tarefa (HOLDERBAUM, 2009; HOLDERBAUM; SALLES, submetido).

No grupo de chineses foi aplicado o CELPE-BRAS - Certificado de Proficiência de Português para Estrangeiros. Este exame, desenvolvido pelo Ministério da Educação, com quatro níveis - intermediário, intermediário superior, avançado e avançado superior -, é exigido pelas universidades para ingresso em cursos de graduação e pós-graduação (<http://www.inep.gov.br/ celpebras $>$ ). A maior parte da amostra, cerca de $62 \%$, apresentava nível intermediário e a parcela restante, $28 \%$, nível intermediário superior. Três participantes não realizaram o exame. Por fim, foi utilizado na amostra de chineses o teste de consciência fonológica em português (CONFIAS), proposto por Moojen et al. (2003), que avalia consciência de sílaba (síntese, segmentação, identificação sílaba inicial etc.) e consciência de fonema (produção de palavras que iniciam com som dado, identificação de fonema inicial etc.).

Os dados foram analisados de forma descritiva, pela comparação de médias entre grupos, e por correlações (entre o desempenho dos chineses na tarefa de 
consciência fonológica com TR, porcentagem de erros e magnitude do efeito de priming semântico na tarefa de decisão lexical). Para comparação das médias de TR e de porcentagem de erros nas duas condições (com prime relacionado e com prime não relacionado) em cada grupo, utilizou-se testes t para medidas repetidas. As diferenças entre as magnitudes de efeito de priming semântico dos grupos foram avaliadas através de testes t para amostras independentes.

\section{Resultados}

A Tabela 1 apresenta o desempenho dos grupos na tarefa de decisão lexical no paradigma de priming semântico. Os três grupos apresentaram TRs mais rápidos na condição prime relacionado quando comparado à condição prime não relacionado: chineses $(t(31)=-2,532 ; p=0,017)$, brasileiros adultos $(\mathrm{t}(30)=$ $-7,569 ; \mathrm{p}<0,001)$ e crianças $(\mathrm{t}(25)=-6,978 ; \mathrm{p}<0,001)$. Nos três grupos estudados, não houve efeito de priming semântico no que se refere à precisão de respostas (\% de erros). Em relação à magnitude do efeito de priming semântico (TR com prime não relacionado - TR com prime relacionado), não foram encontradas diferenças significativas entre os grupos de chineses e de brasileiros adultos. Porém, a magnitude do efeito de priming semântico foi significativamente maior para crianças do que para os chineses $(\mathrm{t}(56)=-3,512 ; \mathrm{p}=0,001)$.

TABELA 1 - MÉDIA (M) E DESVIO PADRÃO (DP) DOS TEMPOS DE REAÇÃO, EM MS; PORCENTAGEM DE ERROS (\%) E MAGNITUDE DO EFEITO (DIFERENÇA ENTRE AS CONDIÇÕES COM PRIME RELACIONADO E NÃO RELACIONADO - NR - R) POR GRUPO

\begin{tabular}{l|c|c|c|c|c|c|c|c|c}
\hline & \multicolumn{5}{c|}{ Contexto } & & & \\
\hline & \multicolumn{3}{|c|}{ prime relacionado } & \multicolumn{2}{c|}{ prime não relacionado } & \multicolumn{3}{c}{ NR - R } \\
\hline & $\mathrm{M}$ & $\mathrm{DP}$ & $\%$ & $\mathrm{M}$ & $\mathrm{DP}$ & $\%$ & $\mathrm{M}$ & $\mathrm{DP}$ \\
\hline chineses & 1029 & 257 & 9,7 & 1105 & 242 & 7,5 & 76 & $-2,2$ \\
\hline universitários* & 632 & 123 & 2,3 & 708 & 134 & 2,8 & 76 & 0,5 \\
\hline crianças* & 1222 & 248 & 3,4 & 1454 & 238 & 5,1 & 232 & 1,7 \\
\hline
\end{tabular}

Nota: Resultados apresentados e discutidos em Holderbaum e Salles (submetido). 
Além disso, não houve correlação significativa entre os escores no teste de consciência fonológica e as variáveis da tarefa de decisão lexical no paradigma de priming semântico no grupo de chineses.

\section{Discussão}

Os resultados mostram que o grupo de chineses, com níveis intermediário e intermediário superior no exame de proficiência de português para estrangeiros, mesmo não totalmente proficientes na língua portuguesa, apresentaram benefícios do contexto semântico na tarefa de decisão lexical no paradigma de priming semântico na língua portuguesa (L3 para eles), não se diferenciando de forma significativa dos brasileiros de mesma idade e nível de escolaridade (adultos universitários) e das crianças, que também não são totalmente proficientes na leitura (terceiro ano de escolarização formal). Nessas condições, os participantes parecem realmente acessar o significado dos primes apresentados visualmente na língua portuguesa.

A comparação dos efeitos de priming semântico entre os estudantes de $3^{\text {a }}$ série do EF e os universitários pode ser vista em Holderbaum (2009) e Holderbaum e Salles (submetido), não tendo sido objetivo de estudo do presente trabalho. Nos estudos anteriores, constatou-se que crianças apresentaram maior magnitude de efeito de priming semântico do que os adultos. No estudo aqui apresentado, a magnitude de efeito do grupo de chineses não se diferenciou da dos brasileiros universitários, mas foi inferior a das crianças brasileiras. As crianças foram as que tiveram maior benefício da apresentação de primes semanticamente relacionados aos alvos na tarefa de decisão lexical. É importante salientar que, na modalidade oral, as crianças brasileiras são totalmente proficientes, estando em processo de aprendizagem da modalidade escrita. Já os chineses apresentam nível intermediário em ambas as modalidades, oral e escrita, sendo que é possível que eles não tenham em seu léxico mental (nem oral e nem escrito) alguns estímulos do experimento. Nesses casos, esses funcionam como pseudopalavras para os chineses.

Os resultados do presente estudo vão ao encontro dos de Gollan et al. (1997) e Finkbeiner (2003), que demonstraram um efeito de priming na velocidade das respostas, apesar de terem utilizado o paradigma de priming de repetição e não de priming semântico.

Um fator que pode ter facilitado a presença de efeitos de priming semântico no grupo de chineses do presente estudo, mesmo sendo testados em L3, foi o fato de o experimento conter estímulos com forte associação semântica no 
português e a seleção dos estímulos ter sido feita com base em estudo empírico com crianças de terceira série (SALLES; MACHADO; HOLDERBAUM, 2009). Devitto e Burgess (2004) estudaram pares de palavras com fraca associação semântica em tarefa de decisão lexical em inglês e não encontraram efeito de priming nos bilíngues adultos, que foram expostos a uma segunda (inglês) ou terceira língua mais tarde no desenvolvimento.

Apesar de alguns estudos com monolíngues apenas, comparando crianças (grupo menos proficiente) e adultos (grupo mais proficiente), sugerirem que leitores iniciantes, menos proficientes, se beneficiam mais do contexto semântico em tarefa de decisão lexical (SCHWANTES; BOESL; RITZ, 1980; SIMPSON; FOSTER, 1986; SIMPSON; LORSBACH, 1983), o presente estudo com a amostra de multilíngues corrobora parcialmente com tal hipótese. As diferenças encontradas entre adultos brasileiros e chineses, em termos de proficiência na língua portuguesa, não refletiram diferenças em termos de acesso implícito à informação semântica de palavras lidas na tarefa de decisão lexical.

Dentre os modelos explicativos para os efeitos de priming semântico (ver MCNAMARA, 2005, para uma revisão dos modelos), os mais conhecidos são Propagação da Ativação (ANDERSON, 1976, 2004) e Processos Estratégicos (BECKER, 1980) - modelos de expectativa. A propagação da ativação explica melhor os resultados de estudos com SOAs (Stimulus Onset Asynchrony) curtos (NEELY, 1991), quando o estímulo-prime pré-ativa automaticamente as representações de todas as palavras que são semântica ou associativamente relacionadas a ele, facilitando a decisão lexical do alvo. Para SOAs longos, os dados podem ser melhor interpretados pelo modelo de expectativa, através do qual é esperado que o participante use a informação do prime para gerar uma série de potenciais palavras-alvo (CHWILLA; HAGOORT; BROWN, 1998). Caso o alvo seja um dos estímulos pré-ativados, a decisão lexical será mais rápida e mais acurada. A facilitação ocorre quando o alvo apresentado está na lista das palavras esperadas por serem semanticamente relacionadas ao prime (NIEVAS; JUSTICIA, 2004). Como neste estudo foi utilizado um SOA de $500 \mathrm{~ms}$, essa última abordagem teórica estaria mais bem relacionada aos achados.

É interessante notar que não houve relação entre a medida de consciência fonológica, testada em português, e os escores na tarefa de decisão lexical em palavras apresentadas visualmente (acesso ao léxico visual) para o grupo de chineses. Conforme relatado anteriormente, apenas a consciência morfológica prediz o desempenho em leitura em crianças chinesas (MCBRIDE-CHANG et al., 2005). Experimentos com populações com escrita logográfica ${ }^{5}$ (READ et

5 Os logogramas são unidades lexicais que representam palavras e ideias. 
al., 1986) constataram que, por exemplo, amostras de chineses que só tinham aprendido os caracteres logográficos tradicionais da língua foram deficitários com relação às tarefas de consciência fonológica, em comparação aos que tinham aprendido ao mesmo tempo a escrita logográfica e a escrita alfabética (pinyin). Dessa forma, esse tipo de habilidade de processamento fonológico para a leitura em chineses não parece ser mesmo necessário.

\section{Conclusão e Considerações finais}

Os resultados indicam que chineses, assim como os brasileiros, adultos e crianças, apresentaram efeito de priming semântico, respondendo de forma mais rápida aos alvos precedidos por primes semanticamente relacionados. $\mathrm{O}$ acesso lexical na Língua Portuguesa (L3), por parte dos chineses, mostrou facilitação do contexto semântico, facilitação essa semelhante à encontrada nos falantes do português como L1.

Em línguas logográficas como o chinês escrito, o significado das palavras é acessado pela forma visual e não através da decodificação das letras para sons (como nas línguas alfabéticas). O pareamento da fonologia no chinês é no nível da sílaba e não do fonema, portanto, a escrita chinesa não permite uma verdadeira análise fonêmica, que é fundamental na escrita alfabética, sendo as regras de conversão grafema-fonema impossíveis no chinês (TAN; LAIRD; LI; FOX, 2005). É importante salientar que neste estudo a consciência fonológica não foi avaliada na primeira língua (mandarin), mas sim no português (em L3), cujo sistema de escrita (alfabético) é substancialmente regido pelo princípio alfabético.

Limitações deste estudo estão relacionadas a não se ter um escore de leitura de palavras nos chineses para correlacionar aos resultados do priming semântico, além do fato de que não houve controle do vocabulário dos chineses na Língua Portuguesa, ou seja, não se pode garantir que eles conheçam o significado de todos os estímulos contidos no experimento de priming semântico.

Finalmente, este é um tema que ainda precisa ser mais explorado, especialmente no Brasil, e que está em crescente debate internacionalmente, como mostra o editorial da revista Brain \& Language, de 2009 (volume 109), que faz referência a uma área denominada Neurociência do Multilinguismo ("Neuroscience of Multilingualism"). 


\section{REFERÊNCIAS}

ALTARRIBA, J.; BASNIGHT-BROWN, D. M. Methodological considerations in performing semantic - and translation - priming experiments across languages. Behavior Research Methods, v. 39, n. 1, p. 1-18, 2007.

ANDERSON, J. R. Language, memory and thought. Hillsdale, NJ: Elrbaum, 1976.

. Psicologia Cognitiva e suas implicações experimentais. Tradução de: D. C. Alencar. Rio de Janeiro: LTC, 2004.

ANTHONY, J. L.; LONIGAN, C. J. The nature of phonological awareness: Converging evidence from four studies of preschool and early grade school children. Journal of Educational Psychology, v. 96, n. 1, p. 43-55, 2004.

BECKER, A. C. Semantic contexts effects in visual word recognition: An analysis of semantic strategies. Memory and Cognition, v. 8, n. 6, p. 493-512, 1980.

BETJEMANN, R. S.; KEENAN, J. M. Phonological and semantic priming in children with reading disability. Child Development, v. 79, n. 4, p. 1086-1102, 2008 .

BLOOMFIELD, L. Language. New York: Henry Holt, 1933.

CHWILLA, D. J.; HAGOORT, P.; BROWN, C. M. The mechanism underlying backward priming in a lexical decision task: Spreading activation versus semantic matching. Quarterly Journal of Experimental Psychology, v. 51A, n. 3, p. 531-560, 1998.

DEVITTO, Z.; BURGESS, C. Theoretical and methodological implications of language experience and vocabulary skill: Priming of strongly and weakly associated words. Brain and Cognition, v. 55, p. 295-299, 2004.

FINKBEINER, M. Task-dependent L2-L1 translation priming: An investigation of the separate memory systems account. In: COHEN, James; MCALISTER, Kara T.; ROLSTAD, Kellie; MACSWAN, Jeff (Ed.). ISB4: Proceedings of the 4th International Symposium on Bilingualism. Somerville, MA: Cascadilla Press, 2003. p. 741-750. 
FINKBEINER, M.; FORSTER, K. I.; NICOL, J.; NAKAMURA, K. The role of polysemy in masked semantic and translation priming. Journal of Memory and Language, v. 51, p. 1-22, 2004.

FORSTER, K. I. The microgenesis of priming effects in lexical access. Brain and Language, v. 68, p. 5-15, 1999.

FRENCK, C.; PYNTE, J. Semantic representation and surface forms: A look at across language priming in bilinguals. Journal of Psycholinguistic Research, v. 16, p. 383-396, 1987.

GOLLAN, T. H.; FORSTER, K. I.; FROST, R. Translation priming with different scripts: masked priming with cognates and noncognates in Hebrew-English bilinguals. Journal of Experimental Psychology: Learning, Memory and Cognition, v. 23, n. 5, p. 1122-1139, 1997.

GROSJEAN, F. The bilingual as a competent but specific speaker-hearer. Journal of Multilingual and Multicultural Development, v. 6, p. 467-477, 1985.

. The bilingual as a person. In: TITONE, R. (Ed.). On the Bilingual Person. Ottawa: Canadian Society for Italian Studies, 1989.

HO, S. H.; BRYANT, C. E. Development of phonological awareness of Chinese children in Hong Kong. Journal of Psycholinguistic Research, v. 26, n. 1, p. 109-126, 1997.

HOLDERBAUM, C. S. Efeitos de priming semântico em tarefa de decisão lexical com diferentes intervalos entre estímulos. Dissertação (Mestrado) - Programa de Pós-Graduação em Psicologia, Instituto de Psicologia, Universidade Federal do Rio Grande do Sul, 2009.

HOLDERBAUM, C. S.; SALLES, J. F. Semantic priming effects in a lexical decision task: comparing third graders and college students in two different stimulus onset asynchronies. Manuscrito submetido para publicação, 2009.

JIANG, N. Testing processing explanations for the asymmetry in masked cross-language priming. Bilingualism: Language and Cognition, v. 2, n. 1, p. 59-75, 1999.

JIANG, N.; FORSTER, K. I. Cross-language priming asymmetries in lexical decision and episodic recognition. Journal of Memory and Language, v. 4, p. 32-51, 2001. 
KEATLEY, K. W.; SPINKS J. A.; GELDER, B. Asymmetrical cross-language priming effects. Memory \& Cognition, v. 12, n. 1, p. 70-84, 1994.

MARTIN, C. D.; DERING, B.; THOMAS, E. M.; THIERRY, G. Brain potentials reveal semantic priming in both the 'active' and the 'non-attended' language of early bilinguals. Neuroimage, v. 47, p. 326-333, 2009.

MCBRIDE-CHANG, C.; CHO, J-R.; LIU, H.; WAGNER, R. K.; SHU, H.; ZHOU, A. et al. Changing models across cultures: Associations of phonological awareness and morphological structure awareness with vocabulary and word recognition in second graders from Beijing, Hong Kong, Korea, and the United States. Journal of Experimental Child Psychology, v. 92, p. 140-160, 2005.

MCNAMARA, T. P. Semantic priming: Perspectives from memory and word recognition. New York: Psychology Press, 2005.

MOOJEN, S.; LAMPRECHT, R.; SANTOS, R. M.; FREITAS, G. M.; BRODACZ, R.; SIQUEIRA, M.; COSTA, A. C.; GUARDA, E. CONFIAS Consciência Fonológica Instrumento de Avaliação Seqüencial. São Paulo, Casa do Psicólogo, 2003.

MORAIS, J. A arte de ler. São Paulo: Editora da Universidade Estadual Paulista, 1996.

NEELY, J. H. Semantic priming effects in visual word recognition: A selective review of current findings and theories. In: BESNER, D.; HUMPHREYS, G. H. Basic processes in reading, visual word recognition. New Jersey: Lawrence Erlbaum Associates, 1991. p. 264-336

NIEVAS, F.; JUSTICIA, F. A cross-sectional study about meaning access processes for homographs. Cognitive Development, v. 19, p. 95-109, 2004.

PLAUT, D. C.; MCCLELLAND, J. L.; SEIDENBERG, M. S.; PATTERSON, K. Understanding normal and impaired word reading: Computational principles in quasi-regular domains. Psychological Review, v. 103, n. 1, p. 56-115, 1996.

READ, C.; ZHANG, Y.; NIE, H.; DING, B. The ability to manipulate speech sounds depends on knowing alphabetic writing. Cognition, v. 24, p. 31-44, 1986.

SAER, D. J. An inquiry into the effects of bilingualism upon the intelligence of young children. Journal of Experimental Pedagogy, v. 6, p. 232-240, 1922. 
SALLES, J. F.; JOU, G. I.; STEIN, L. M. O paradigma de priming semântico na investigação do processamento de leitura de palavras. Interação em Psicologia, v. 11, n. 1, p. 71-80, 2007.

SALLES, J. F.; MACHADO, L. L; HOLDERBAUM, C. S. Normas de associação semântica de 50 palavras do português brasileiro para crianças: tipo, força de associação e tamanho do conjunto do alvo. Revista Interamericana de Psicologia, v. 43, n. 1, p. 57-67, 2009.

SCHWANTES, F. M.; BOESL, S. L.; RITZ, E. G. Children`s use of context in word recognition: A psycholinguistic guessing name. Child Development, v. 51, p. 730-736, 1980.

SEIDENBERG, M. S.; MCCLELLAND, J. L. A distributed, developmental model of word recognition and naming. Psychological Review, v. 97, p. 477-452, 1989.

SIMPSON, G. B.; FOSTER, M. R. Lexical Ambiguity and Children`s Word Recognition. Developmental Psychology, v. 22, n. 2, p. 147-154, 1986.

SIMPSON, G. B.; LORSBACH, T. C. The development of automatic and conscious components of contextual facilitation. Child Development, v. 54, p. 760-772, 1983.

STACKHOUSE, J. Phonological awareness: Connecting speech and literacy problems. In: HODSON, B.; EDWARDS, M. L. (Eds.). Perspectives in Applied Phonology, Gaithersburg, MD: Aspen Publications, p. 157-196, 1997.

STAHL, S. A.; MURRAY, B. A. Defining phonological awareness and its relationship to early reading. Journal of Educational Psychology, v. 86, n. 2, p. 221-234, 1994.

TAN, L. H.; LAIRD, A. R.; LI, K.; FOX, P. T. Cognitive neuroanatomical correlates of phonological processing of Chinese characters and alphabetic words: A meta-analysis. Human Brain Mapping, v. 25, p. 83-91, 2005.

Texto recebido em 25 de junho de 2010.

Texto aprovado em 3 de agosto de 2010. 